\title{
Small Bursts of Frequent Communications-an Effective Communication Method in a Busy Emergency Department
}

\author{
Akshay Kumar $^{1} \cdot$ Richa Aggarwal ${ }^{2} \cdot$ Sanjeev Bhoi ${ }^{3} \cdot$ Ajay Sharma ${ }^{4,5,6}$
}

Published online: 18 November 2019

(C) Association of Surgeons of India 2019

Communication skills for a caregiver, medical as well as nursing, are of utmost importance in providing quality emergency care. The Institute of Medicine in 2001 described "patient centered care" as one of the pillars of quality in healthcare. Effective communication between the patient and provider is integral to patient centered care [1]. Patient and family satisfaction are directly related to the quality of timely and effective communication between clinician and patient's family [2-9]. The most common reasons for litigations and complaints in any leading medical center are related to poor communication and inappropriate conduct of healthcare givers rather than perceived sub-optimal treatment. There is no doubt that government medical facilities are often overwhelmed at all levels of care. The situation is further compounded by the lack of formal competence-based training in human factors. A multitude of reasons such as high volume of critically sick patients, perpetual shortage of trained staff, lack of availability of senior personnel especially at odd hours and inadequate facilities at primary and secondary levels make most emergency departments a challenging area to provide adequate communication consistently.

Time is always at premium. Medical and nursing staff in a tertiary care emergency department (ED) are always over-

Akshay Kumar

akshay2111@gmail.com

1 Department of Emergency Medicine, All India Institute of Medical Sciences, New Delhi, India

2 Department of Critical and Intensive Care, JPN Apex Trauma Centre, All India Institute of Medical Sciences, New Delhi, India

3 Department of Emergency Medicine, JPN Apex Trauma Centre, All India Institute of Medical Sciences, New Delhi, India

4 Wirral Teaching Hospital, Wirral, UK

5 University of Liverpool, Liverpool, UK

6 Surgical Training Merseyside, Northwest of England, Merseyside, UK stretched and over-burdened. In such settings, it becomes all the more important to provide information that is accurate, valid, razor sharp, empathetic, considerate and crisp. A timely and efficient process of communication has to be evolved for such scenario especially in emergency which is often managed by resident doctors. It should not appear as a distraction by doctors and nurses from the vital clinical tasks that they must perform. The communication skills are all the more relevant in a busy ED especially in low- and middle-income countries, where any illness is a social event and huge crowd can visit hospital at the time of emergency. The need for an effective crisis communication strategy can be highlighted in the following four points. Firstly, the patient is often acutely and critically sick, leaving little time for a detailed explanation. Secondly, the situation of the patient is often rapidly changing that require more frequent monitoring and interventions. Thirdly, the diagnosis is often not clear during the primary survey when a patient is being assessed and simultaneously resuscitated on the basis of physiological derangements. On top of all these, the patients and their accompanying family members are, not surprisingly, extremely anxious. The atmosphere is often so charged that the reports of violence and skirmishes are common occurrences. An observational study from Australia demonstrated the need for better training in communication for emergency personnel as shown by the analysis of 1286 distinct communication episodes [10]. A number of exemplary consultants/attendings set up an example for resident doctors and interns in purposeful and effective communication. However, not everyone is so lucky to have worked in a clinical department that provides opportunity to learn communication skills. Though there are individuals who have inherent power of communication but for the majority health care providers there is an urgent need for structured mandatory training in communication skills across all disciplines. The authors believe that the development of good quality communication skills would not only improve patient care and family satisfaction but also enhance the job satisfaction of doctors and nurses working in the emergency settings. Having 
a happy and satisfied client, customer or patient is a matter of self-pride and extreme satisfaction.

Unlike several clinical situations for example breaking news for cancer, there are no established models for providing an effective communication between care provider and patient/relatives in an ED, a place where it is crucially important to accomplish smooth functioning. The Society of Academic Emergency Medicine identified 5 essential stepwise components of communication in an ED: establishing rapport, gathering information, giving information, providing comfort (empathy) and ensuring collaboration [11]. They described the key steps of an effective ED communication but did not provide a well-defined structure to this tool such as the SPIKES model. The full form of this abbreviation is: $S$ - Setting up the scene, $P$-perception of patient and relatives in a given situation, I-Invitation to disclose, $K$ - sharing the information, E- Empathise, S- Strategy and Summary. This was first described by Baile et al in 2000 as a communication protocol in cancer patients. A six-step structured protocol for breaking bad news to the family is an excellent step-by-step approach to address this challenge [12]. However, in a high-volume ED in a government-run teaching hospital of a developing country, this approach, we felt, needs to be modified and finetuned. SPIKES model of communication works well when there is a good rapport between the treating team and patient and family that enables to address the full spectrum of patient's concerns [12]. In emergency, the doctor usually meets the patient and family for the first time and the management decisions have to be made expeditiously without complete diagnostic information. The purpose of communication in ED setting is not about diagnosis; it is more about caring and sharing the information. This paradigm shift in focus of communication becomes all the more important in a lifethreatening condition. The relatives in ED may not be so keen on the possible diagnosis but more focused on what the immediate plan of life-saving measures are, and if these are being implemented in a timely fashion. There are times when there may be a discrepancy in what is seen versus what the relatives believe should be instituted. That specific comparison does occupy their mind until their patient comes out of the lifethreatening spiral of events leading to possible death or disability. The plan of management includes providing information on what point-of-care testing is being planned, the treatment/procedure being instituted and if there is any specialist reviews their sick relative is awaiting. The objectives of treating team are no different, i.e. the diagnosis can wait while the patient is being resuscitated. The lack of a diagnosis should not come in the way of having enough confidence to express a range of possibilities (i.e. differential diagnosis) even if it means stating, "we do not have a clear diagnosis yet, however, we are trying our best". The key point is the relatives need to be convinced that "the treating team's best will be good enough for their patient to survive".
Based on our usual yet-unreported and well-tested approach, we suggest "short bursts of frequent communications". The scene could be set up in one (relatively) isolated corner of an ED that may mean walking out of the area of action (i.e. resuscitation) that is often quite a busy area. The first step includes introducing self and establishing rapport, briefly asking for what the family and relatives know. Then, it is suggested that the care provider should make an eye contact and gauge body language about what they [patient relatives] want to know, if not clear, ask directly in a sympathetic manner. The care provider, often the team leader of resuscitation who peels off for a short duration, simultaneously gathers necessary clues to the course of events and uses this opportunity to provide short burst of information on what has been done, what is currently going on (management wise, for example that the patient has been started on high flow oxygen and intravenous fluids) and what will be the further plan of assessment (e.g. the patient will be transferred shortly for an abdominal X-ray and is awaiting a review by the general surgeon). At the next step, the relatives are given a minute or so to digest the gravity of situation and are provided short verbal assurance and empathy. At this point, the treating team invites 1-2 family members or relatives to walk with the doctor or nurse to the side of the patient's bed for them to actually watch what is going on during the secondary survey, i.e. when the patient is stabilized and detailed assessment is being performed that often requires lesser number of professionals. This invite fosters the confidence in the treating team and assures them by helping to build an image of treating team that is engrossed in providing standard care. The care provider can leave the relatives/family at the bedside and tell them that he or she will return to talk after sometime if they have any further queries or needs while he/she is busy doing important tasks specific to the index patient. The entire episode takes about $2-3 \mathrm{~min}$. The care provider should ideally return within 15-20 min for another brief episode of communication with the patient's family in order to update them about the progress or lack of it. "Role-play" can be used as a training model in simulated setting for developing communication skills that are necessary for managing acutely unwell patients.

To conclude, in a high-volume ED, it is crucial for doctors and nurses to be endowed with skills of "short bursts of frequent communication" to accomplish an efficient, safe and patient centered care for all.

\section{References}

1. Institute of Medicine (USA) (2001) Crossing the quality chasm: a new health system for the 21st century. Br Med J 323:1192

2. Taylor C, Berger JR (2004) Patient satisfaction in Emergency Medicine. Emerg Med J 21:528-532

3. Taylor DM, Wolfe R, Cameron PA (2002) Complaints from emergency department patients largely result from treatment and communication problems. Emerg Med J 14:43-49 
4. Hunt MT, Glucksman ME (1991) A review of 7 years of complaints in an inner-city accident and emergency department. Arch Emerg Med 8:17-23

5. Kadzombe EA, Coals J (1992) Complaints against doctors in an accident and emergency department: A 10-year analysis. Arch Emerg Med 9:134-142

6. Sun BC, Adams J, Orav EJ, Rucker DW, Brennan TA, Burstin HR (2000) Determinants of patient satisfaction and willingness to return with emergency care. Ann Emerg Med 35:426-434

7. Schwartz LR, Overton DT (1987) Emergency department complaints: A one-year analysis. Ann Emerg Med 16:857-861

8. Bursch B, Beezy J, Shaw R (1993) Emergency department satisfaction: What matters most? Ann Emerg Med 22:586-591

9. Boudreaux ED, Ary RD, Mandry CV, McCabe B (2000) Determinants of patient satisfaction in a large municipal ED: the role of demographic variables, visit characteristics and patient perceptions. Am J Emerg Med 18:394-400

10. Coiera EW, Jayasuriya R, Hardy J, Bannan A, Thorpe MEC (2002) Communication loads on clinical staff in the emergency department. Med J Aust 176:415-418

11. Knopp R, Rosenzweig S, Bernstein E et al (1996) Physician-patient communication in the emergency department, part 1. Acad Emerg Med 3:1065-1069

12. Baile WF, Buckman R, Lenzi R, Glober G, Beale E, Kudelka AP (2000) SPIKES - A six step protocol for delivering bad news: application to the patient with cancer. Oncologist 5:302-311

Publisher's Note Springer Nature remains neutral with regard to jurisdictional claims in published maps and institutional affiliations. 